\section{Avaliação da atenção ao parto por adolescentes em um hospital universitário}

\section{Evaluation of birthing care provided to adolescents in a university hospital}

Cleci de Fátima Enderle 1

Nalú Pereira da Costa Kerber 2

Lulie Rosane Odeh Susin 3

Raúl A. Mendoza-Sassi 4

1-3 Grupo de Pesquisa Viver Mulher do Programa. Pós-Graduação em Enfermagem. Universidade Federal do Rio Grande. Rua Gen. Osório. s.n. Centro. Rio Grande, RS, Brasil. CEP: 96.200-190.

E-mail: clecienderle@gmail.com

4 Faculdade de Medicina (FAMED). Rio Grande, RS, Brasil.

\begin{abstract}
Objectives: evaluate birthing care from the perspective of adolescents seen at a university hospital.

Methods: a quantitative descriptive study developed at a university hospital in the south of Brazil. The research sample was comprised of 269 adolescents, between 10 and 19 years of age, from July 2008 to October 2009. The data was collected through interviews about the practices of vaginal childbirth. Statistical analysis was performed using Fisher's Exact Test. The fin-dings were then compared with the recommended practices for perinatal care recommended by the Ministry of Health (MH).

Results: the lithotomic position was adopted in 99\% of cases; an episiotomy was performed on $89.6 \%$ of delivering women; digital vaginal examinations were conducted by more than one examiner in $69.4 \%$ of deliveries; routine preventive venous catheterization was performed in $91.8 \%$ of adolescent girls and food and water restrictions were put in place in $87.4 \%$ of cases. These findings are not consistent with $\mathrm{MH}$ guidelines for birthing care.

Conclusions: many of the guidelines recommended by MH for birthing care are disregarded at the reviewed institution and must be revised in order to address the directives of the Ministry of Health.
\end{abstract}

Key words Adolescent, Parturition, Pregnancy in adolescence, Normal childbirth

\title{
Resumo
}

Objetivos: avaliar a atenção ao parto na ótica de adolescentes assistidas em um hospital universitário.

Métodos: estudo quantitativo, descritivo, desenvolvido em um hospital universitário do Sul do Brasil. A amostra foi 269 adolescentes, entre 10 e 19 anos, de julho de 2008 a outubro de 2009. A coleta de dados foi com entrevista acerca das práticas de parto normal. Para análise estatística, foi utilizado o Teste Exato de Fisher. Os achados foram comparados com as práticas recomendadas à atenção perinatal preconizados pelo Ministério da Saúde (MS).

Resultados: a posição litotômica adotada em $99 \%$ dos casos; a episiotomia foi realizada em $89,6 \%$ das parturientes; os toques vaginais foram realizados por mais de um examinador em $69,4 \%$ dos partos; a cateterização venosa profilática de rotina foi instalada em 91,8\% das adolescentes e a restrição hídrica e alimentação esteve presente em 87,4\%. Estes achados não condizem com as orientações preconizadas pelo MS acerca da atenção ao parto.

Conclusões: muitas condutas recomendadas pelo MS para o parto são desconsideradas na instituição em estudo, precisando ser revistas de modo a contemplar as diretrizes do $M S$.

Palavras-chave Adolescente, Parto, Gravidez na adolescência, Parto normal 


\section{Introdução}

A gravidez na adolescência é uma situação de risco psicossocial que pode ser reconhecida como um problema para os jovens, que assim iniciam uma família não intencionada, sendo um período de transformação profunda no corpo, na mente e na forma de relacionamento social do indivíduo. ${ }^{1}$

As adolescentes merecem um olhar singular durante a assistência pré-natal, pois representam a categoria de mais baixa aderência ao serviço (menor número de consultas e maior índice de nãocomparecimento). ${ }^{2}$ Conforme estudo ${ }^{3}$ de base populacional realizado em Rio Grande no ano de 2007, foi encontrada cobertura de pré-natal de $96 \%$, com uma média de 7,4 consultas e média de idade das gestantes de 25,6 anos, com as adolescentes (19 anos ou menos) totalizando $20,2 \%$ do total dos atendimentos.

Considerando a adolescência como um período repleto de mudanças significativas, a maioria dos estudos aborda a gestação, parto e puerpério com enfoque nos riscos físicos, psicológicos e sociais, sem enfatizar os aspectos relacionados com a assistência ao ciclo gravídico-puerpeal.4,5 Receber atenção adequada no momento do parto pode ser um passo decisivo e importantíssimo para amenizar os agravos que podem advir de uma gravidez precoce.

Dessa forma, torna-se claro que as adolescentes grávidas precisam de um olhar cuidadoso por parte das autoridades e gestores em saúde. O Ministério da Saúde (MS), buscando uma assistência mais humanizada, o que envolve um conjunto de práticas que visam à promoção do parto e do nascimento saudáveis e a prevenção da morbi-mortalidade materna e perinatal, instituiu em junho de $2000 \mathrm{o}$ Programa de Humanização no Pré-Natal e Nascimento (PHPN), através da Portaria GM n ${ }^{\circ}$ 569.6

O PHPN tem como principal objetivo assegurar a melhoria do acesso, da cobertura e da qualidade do acompanhamento pré-natal, da assistência ao parto e puerpério às gestantes e ao recém-nascido, na perspectiva dos direitos de cidadania. 6 O MS incorporou em seus manuais técnicos algumas recomendações que, segundo a Organização Mundial da Saúde (OMS), são essenciais para a atenção prénatal, perinatal e puerperal. Tais recomendações assentaram-se em revisão sistemática de estudos controlados e na aplicação dos conceitos da medicina baseada em evidências. ${ }^{7}$

Os dez princípios fundamentais da atenção perinatal, assinalados pela OMS,7 indicam que o cuidado na gestação e no parto normal deve contemplar as seguintes recomendações: não ser medicalizado; ser baseado no uso de tecnologia apropriada; ser baseado em evidências; ser regionalizado e baseado em sistema eficiente de referência; ser multidisciplinar e multiprofissional; ser integral; estar centrado nas famílias; ser apropriado, tendo em conta as diferentes pautas culturais; compartilhar a tomada de decisão com as mulheres; respeitar a privacidade, a dignidade e a confidencialidade das mulheres. ${ }^{2}$ Tais recomendações estão sendo incorporadas nos materiais técnicos, bem como nas ferramentas de monitoração e avaliação da OMS.

A partir destas recomendações as práticas de assistência ao parto normal passaram a ser classificadas em: práticas claramente úteis e que devem ser incentivadas; práticas prejudiciais ou ineficazes e que devem ser eliminadas; práticas com evidência insuficiente para apoiar uma recomendação e que deveriam ser usadas com precaução; práticas frequentemente utilizadas de forma inapropriada, provocando mais dano que benefício. 7

Esse conjunto de evidências tem sido utilizado por muitos hospitais públicos do Brasil no intuito de oferecer uma assistência humanizada ao parto e, muitos o têm conseguido, provando ser possível, por exemplo, reduzir as taxas de cesárea, reduzir a utilização de mecanismos de indução e o aceleramento do parto, incentivar o aleitamento materno, o uso criterioso de amniotomia e a episiotomia, entre outros. 8

Porém, também são encontrados estudos divergentes, como por exemplo, o que foi realizado em Botucatu, São Paulo, que destaca o fato de que apesar de ser percebido tendência à humanização e incorporação de evidências científicas na assistência, algumas práticas demonstradamente úteis no parto ainda são pouco implementadas na maternidade estudada, enquanto outras prejudiciais ou ineficazes continuam sendo realizadas, supostamente por tratarse de serviço terciário. ${ }^{9}$

Ao entender a atualidade ainda da temática e a necessidade dos serviços de atenção obstétrica seguirem as recomendações preconizadas, o presente estudo tem a seguinte questão de pesquisa: Como estão sendo desenvolvidas em um hospital universitário as práticas recomendadas pela OMS/MS para o parto? Por entender que a adolescência é um período delicado e que a qualidade da atenção ao parto pode ser decisiva no processo de transição para a vida adulta, o objetivo deste estudo foi avaliar a atenção ao parto sob a ótica das adolescentes assistidas em um hospital universitário. 


\section{Métodos}

Este foi um estudo derivado de uma pesquisa multicêntrica, de caráter quantitativo, descritivo, desenvolvido em um hospital universitário (HU) do sul do Brasil, que integra o patrimônio da União e a rede de serviços que compõe o Sistema Único de Saúde (SUS), constituindo-se em campo de estágio para diferentes cursos de graduação da área da saúde.

O contexto investigativo do presente estudo foi o Centro Obstétrico (CO), do $\mathrm{HU}$, com área física composta por: sala para consulta, sala para exame, sala de pré-parto com três leitos, sala de parto, sala para reanimação do recém-nascido, banheiro, expurgo e copa.

A amostra estudada constituiu-se de 269 adolescentes, entre 10 e 19 anos, que tiveram seu processo de parturição pelo SUS no centro obstétrico do HU, no período de julho de 2008 a outubro de 2009. Nesse período foram realizados 2034 partos pelo SUS, destes $360(17,7 \%)$ eram de adolescentes. Dessas adolescentes, 65 (18,1\%) foram excluídas por não terem passado pelo centro obstétrico, indo direto ao bloco cirúrgico, ou por terem realizado internação particular ou convênio, e com feto morto. Ocorreram três perdas por atraso dos coletadores de dados e 23 recusas.

As adolescentes foram submetidas à entrevista individual estruturada, conduzidas por acadêmicas de enfermagem previamente treinadas, na unidade de internação obstétrica, nas primeiras 24 horas após o parto, visando obter as respostas o mais próximo possível da assistência recebida, evitando viés de memória, visando que o depoimento não fosse influenciado pela alegria e emoção do nascimento, ou pelo desgaste sofrido pela paciente.

Foram pesquisadas as variáveis sociodemográficas (escolaridade, renda e classe econômica), dados pessoais (idade e cor da pele), e dados específicos: realização de pré-natal; atendimento considerado ideal (realização das práticas consideradas pelas OMS/MS como claramente úteis e que devem ser incentivadas); recebimento de orientações a respeito do parto; respeito à privacidade no atendimento; avaliação do conhecimento de seus direitos; relação da adolescente com a equipe de trabalho; realização de tricotomia e enema; alimentação; higiene; episiotomia; uso da soroterapia profilática; tipo de parto; presença de acompanhante; orientações recebidas pela da equipe, e toque vaginal.

Para a avaliação da atenção ao parto pelas adolescentes, foram identificadas as condutas e rotinas adotadas no centro obstétrico e comparandoas com as práticas recomendadas à atenção perinatal, assinalados pela OMS, e preconizados pelo MS. Foi utilizado o teste exato de Fisher, para comparação das proporções das variáveis categóricas, buscando identificar os fatores associados ao atendimento considerado ideal ao parto e o trabalho da equipe ter influenciado no atendimento.

O nível de significância estatística considerado nas análises foi de 5\%. Após a codificação das variáveis, foi criado um banco de dados no programa Epi-Info versão 6.04 (Center for Disease Control and Prevention, Atlanta, EUA), sendo realizada dupla digitação, por digitadores independentes, em ordem inversa. Para análise estatística, foi utilizado o pacote Stata versão 10.0 (StataCorp, College Station, EUA).

As adolescentes e seus representantes legais foram esclarecidos sobre a pesquisa e, estando de acordo, assinaram o Termo de Consentimento Livre Esclarecido. O estudo multicêntrico do qual se origina este estudo foi aprovado pelo parecer $n^{\circ}$ 31/2008, emitido pelo Comitê de Ética em Pesquisa na Área da Saúde da Universidade Federal do Rio Grande.

\section{Resultados}

Conforme a Tabela 1 que registra os dados referentes às características demográficas e socioeconômicas, a idade predominante das adolescentes foi de 18 e 19 anos $(27,9 \%)$, a maioria com 5 a 8 anos de estudo $(59,1 \%)$ e de cor branca $(59,4 \%)$ e da classe C com $(62,7 \%)$

Quanto às práticas realizadas no parto normal (Tabela 2), observa-se que apenas $5,6 \%$ das adolescentes tiveram o acompanhamento de algum familiar ou amigo no centro obstétrico. De acordo com $84 \%$ das adolescentes, a privacidade e a intimidade durante a permanência no centro obstétrico foram respeitadas. Quando perguntadas se conheciam seus direitos enquanto parturientes, somente $6,7 \%$ firmaram conhecer, o que diminui o poder de reivindicar e argumentar, mesmo tendo essa prerrogativa assegurada por lei. 10

Foram oferecidas informações sobre o parto (como este iria acontecer, os períodos de dilatação, e o que elas poderiam fazer para colaborar) para $37,3 \%$ das adolescentes.

Como achado em outras questões analisadas, foram oferecidas orientações sobre formas de relaxamento a realizar na sala de pré-parto (técnicas de respiração, manter a posição de decúbito lateral esquerdo, tomar banho morno e fazer força no momento certo do parto) para $56,0 \%$ das adolescentes e $39,0 \%$ das gestantes tomaram banho ou realizaram higienização. 


\section{Tabela 1}

Descrição da amostra de acordo com características demográficas e socioeconômicas (N=269). Rio Grande, RS. 2008-2009.

\begin{tabular}{|c|c|c|}
\hline Características & $\mathbf{N}$ & $\%$ \\
\hline \multicolumn{3}{|l|}{ Idade (anos) } \\
\hline 12 & 1 & 0,4 \\
\hline 14 & 12 & 4,5 \\
\hline 15 & 20 & 7,4 \\
\hline 16 & 46 & 17,0 \\
\hline 17 & 40 & 14,9 \\
\hline 18 & 75 & 27,9 \\
\hline 19 & 75 & 27,9 \\
\hline \multicolumn{3}{|l|}{ Cor da pele } \\
\hline Branca & 160 & 59,4 \\
\hline Morena & 83 & 30,9 \\
\hline Preta & 26 & 9,7 \\
\hline \multicolumn{3}{|l|}{ Anos de estudo } \\
\hline 0 a 4 & 29 & 10,8 \\
\hline 5 a 8 & 159 & 59,1 \\
\hline$\geq 9$ & 81 & 30,1 \\
\hline \multicolumn{3}{|l|}{ Com quem mora } \\
\hline Mãe e/ou pai com marido/companheiro & 35 & 13,1 \\
\hline Mãe e/ou pai sem marido/companheiro & 71 & 26,5 \\
\hline Só com companheiro & 105 & 39.0 \\
\hline Companheiro e sua família & 38 & 14,0 \\
\hline Outro & 20 & 7,4 \\
\hline \multicolumn{3}{|l|}{ Classe econômica* } \\
\hline A & 2 & 0,8 \\
\hline B & 57 & 21,3 \\
\hline $\mathrm{C}$ & 169 & 62,7 \\
\hline$D$ & 35 & 12,9 \\
\hline $\mathrm{E}$ & 6 & 2,3 \\
\hline
\end{tabular}


Práticas utilizadas no parto normal, sob a ótica das adolescentes no Hospital Universitário da Universidade Federal do Rio Grande. (N=269). Rio Grande, RS - 2008-2009.

\begin{tabular}{|c|c|c|}
\hline Características & $\mathbf{N}$ & $\%$ \\
\hline \multicolumn{3}{|c|}{ Tiveram acompanhantes } \\
\hline Sim & 15 & 5,6 \\
\hline Não & 254 & 94,4 \\
\hline \multicolumn{3}{|c|}{ Receberam orientações e informações } \\
\hline Sim & 100 & 37,3 \\
\hline Não & 169 & 62,7 \\
\hline \multicolumn{3}{|c|}{ Tomaram banho (relaxamento e higienização) } \\
\hline Sim & 104 & 39,0 \\
\hline Não & 165 & 61,0 \\
\hline \multicolumn{3}{|c|}{ Conhecimento dos seus direitos } \\
\hline Sim & 18 & 6,7 \\
\hline Não & 251 & 93,3 \\
\hline \multicolumn{3}{|c|}{ Privacidade e intimidade respeitadas } \\
\hline Sim & 226 & 84,0 \\
\hline Não & 43 & 16,0 \\
\hline \multicolumn{3}{|c|}{ Orientações sobre forma de relaxamento } \\
\hline Sim & 151 & 56,0 \\
\hline Não & 118 & 44,0 \\
\hline \multicolumn{3}{|c|}{ Contato precoce com o bebê } \\
\hline Sim & 173 & 64,3 \\
\hline Não & 96 & 35,7 \\
\hline \multicolumn{3}{|c|}{ Bebê mamou antes da primeira hora de vida } \\
\hline Sim & 239 & 89,0 \\
\hline Não & 30 & 11,0 \\
\hline \multicolumn{3}{|c|}{ Realização de tricotomia } \\
\hline Sim & 88 & 32,8 \\
\hline Não & 181 & 67,2 \\
\hline \multicolumn{3}{|c|}{ Realização de enema } \\
\hline Sim & 36 & 13,7 \\
\hline Não & 233 & 86,3 \\
\hline \multicolumn{3}{|c|}{ Realização de episiotomia } \\
\hline Sim & 241 & 89,6 \\
\hline Não & 28 & 10,4 \\
\hline \multicolumn{3}{|c|}{ Toques vaginais feitos por mais de um examinador } \\
\hline Sim & 187 & 69,4 \\
\hline Não & 82 & 30,6 \\
\hline \multicolumn{3}{|c|}{ Parto realizado em posição litotômica } \\
\hline Sim & 267 & 99,0 \\
\hline Não & 2 & 1,0 \\
\hline \multicolumn{3}{|c|}{ Cateterização venosa profilática de rotina } \\
\hline Sim & 246 & 91,8 \\
\hline Não & 22 & 8,2 \\
\hline \multicolumn{3}{|c|}{ Restrição hídrica e alimentar } \\
\hline Sim & 235 & 87,4 \\
\hline Não & 34 & 12,6 \\
\hline
\end{tabular}


Ainda, em relação aos procedimentos e rotinas adotados pela equipe no atendimento ao parto, a tricotomia foi realizada em $32,8 \%$ das pacientes; o enema por $13,7 \%$ das parturientes; posição litotômica em 99,0\% dos casos; episiotomia em $89,6 \%$. Os toques vaginais realizados por mais de um examinador em $69,4 \%$ dos partos; a cateterização venosa profilática de rotina, $91,8 \%$ das adolescentes e a restrição hídrica e alimentação em 87,4\%.

Foi possível perceber que o tipo de parto predominante foi o vaginal, presente em $58,7 \%$ dos casos e dentre estes, $92,4 \%$ das adolescentes referiu ter sido esta sua preferência.

Outros aspectos que ainda se pode visualizar na Tabela 2 refere-se ao contato precoce das adolescentes com seus filhos, com $64,3 \%$ destas tendo recebido o bebê logo que ele nasceu, e a primeira mamada ocorrendo antes de uma hora de vida para $89,0 \%$ dos bebês.

Em relação à assistência recebida no centro obstétrico, 65,1\% das adolescentes afirmaram ter recebido uma assistência ideal. Os fatores que se associaram significativamente a essa opinião foram a boa/ótima relação da equipe com a adolescente e seus familiares, o respeito à privacidade e à intimidade durante o tempo de permanência no centro obstétrico e a instrução recebida sobre formas de relaxamento na sala de pré-parto (Tabela 3). Entre as adolescentes que afirmaram não ter recebido a assistência ideal $(34,9 \%)$, os motivos mais citados foram: falta de atenção, solidão, dor, medo, impaciência da equipe e demora no atendimento.

Outro aspecto avaliado, a influência do trabalho desenvolvido pela equipe, na opinião de $79,5 \%$ das adolescentes, este influiu positivamente no parto. Os fatores significativamente associados a essa opinião foram: a boa/ótima relação da equipe com a adolescente e seus familiares; a adolescente ter sido instruída sobre formas de relaxamento na sala de pré-parto, e a realização do parto vaginal (Tabela 4).

Tabela 3

Fatores associados ao atendimento considerado ideal ao parto no centro obstétrico, de acordo com as adolescentes. ( $N=175)$. Rio Grande, RS, 2008-2009.

\begin{tabular}{|c|c|c|c|}
\hline Variável & $\mathbf{N}$ & $\%$ & $p^{*}$ \\
\hline Idade (anos) & & & 0,19 \\
\hline $12-15$ & 24 & 71,3 & \\
\hline $16-17$ & 49 & 57,3 & \\
\hline $18-19$ & 102 & 68,1 & \\
\hline Classe econômica** & & & 0,67 \\
\hline$A / B$ & 40 & 67,9 & \\
\hline C & 111 & 65,4 & \\
\hline $\mathrm{D} / \mathrm{E}$ & 24 & 58,8 & \\
\hline Recebeu orientações sobre o parto & & & 0,35 \\
\hline Sim & 69 & 76,6 & \\
\hline Não & 106 & 61,8 & \\
\hline Sabia os direitos da parturiente & & & 0,80 \\
\hline Sim & 11 & 61,1 & \\
\hline Não & 164 & 65,4 & \\
\hline Relação da equipe com a adolescente e seus familiares & & & $<0,001$ \\
\hline Muito boa/ótima & 64 & 82,4 & \\
\hline Boa & 102 & 73,1 & \\
\hline Ruim/regular & 9 & 18,4 & \\
\hline Privacidade e intimidade respeitadas no centro obstétrico & & & $<0,001$ \\
\hline Sim & 160 & 70,9 & \\
\hline Em parte & 9 & 42,9 & \\
\hline Não & 6 & 28,6 & \\
\hline Instrução sobre formas de relaxamento no pré-parto e parto & & & 0,01 \\
\hline Sim & 110 & 72,3 & \\
\hline Não & 65 & 55,1 & \\
\hline
\end{tabular}


Fatores associados ao atendimento considerado ideal ao parto no centro obstétrico, de acordo com as adolescentes $(\mathrm{N}=175)$. Rio Grande, RS, 2008-2009.

\begin{tabular}{|c|c|c|c|}
\hline Variável & $\mathbf{N}$ & $\%$ & $p^{*}$ \\
\hline Alimentação durante permanência no centro obstétrico & & & 1,00 \\
\hline Sim & 153 & 65,6 & \\
\hline Não & 22 & 65,0 & \\
\hline Banho ou higienização no centro obstétrico & & & 0,14 \\
\hline Sim & 62 & 59,2 & \\
\hline Não & 113 & 68,6 & \\
\hline Tipo de parto & & & 0,29 \\
\hline Vaginal & 107 & 68,0 & \\
\hline Cesáreo & 68 & 61,0 & \\
\hline Toque vaginal foi feito por mais de um examinador & & & 0,78 \\
\hline Sim & 119 & 63,7 & \\
\hline Não & 54 & 65,8 & \\
\hline Foi feito episiotomia no parto & & & 0,59 \\
\hline Sim & 162 & 67,1 & \\
\hline Não & 13 & 46,5 & \\
\hline Teve acompanhante/familiar/amigos no centro obstétrico & & & 1,00 \\
\hline Sim & 10 & 66,7 & \\
\hline Não & 165 & 65,0 & \\
\hline Identificou as pessoas que prestaram atendimento & & & 0,53 \\
\hline Sim & 145 & 63,3 & \\
\hline Não & 26 & 72,7 & \\
\hline Não sabe & 4 & 80,0 & \\
\hline Recebeu o bebê assim que nasceu & & & 0,59 \\
\hline Sim & 110 & 63,9 & \\
\hline Não & 65 & 67,4 & \\
\hline Bebê mamou no centro obstétrico ou sala de recuperação & & & 0,68 \\
\hline Sim & 154 & 64,8 & \\
\hline Não & 21 & 67,0 & \\
\hline
\end{tabular}


Fatores associados ao fato das adolescentes considerarem que o trabalho desenvolvido pela equipe influenciou no parto (N=214). Rio Grande, RS, 2008-2009.

\begin{tabular}{|c|c|c|c|}
\hline Variável & $\mathbf{N}$ & $\%$ & $p^{*}$ \\
\hline Idade (anos) & & & 0,47 \\
\hline $12-15$ & 33 & 87,9 & \\
\hline $16-17$ & 59 & 79,3 & \\
\hline $18-19$ & 122 & 77,8 & \\
\hline Classe econômica** & & & 0,23 \\
\hline$A / B$ & 46 & 77,6 & \\
\hline $\mathrm{C}$ & 139 & 82,3 & \\
\hline $\mathrm{D} / \mathrm{E}$ & 29 & 70,3 & \\
\hline Recebeu orientações sobre o parto & & & 1,00 \\
\hline Sim & 80 & 79,6 & \\
\hline Não & 134 & 79,1 & \\
\hline Sabia os direitos da parturiente & & & 0,38 \\
\hline Sim & 13 & 72,2 & \\
\hline Não & 201 & 80,1 & \\
\hline Relação da equipe com a adolescente e seus familiares & & & 0,003 \\
\hline Muito boa/ótima & 67 & 84,6 & \\
\hline Boa & 116 & 83,2 & \\
\hline Ruim/regular & 31 & 60,4 & \\
\hline Privacidade e intimidade respeitadas no centro obstétrico & & & 0,14 \\
\hline Sim & 184 & 81,4 & \\
\hline Em parte & 16 & 73,7 & \\
\hline Não & 14 & 65,0 & \\
\hline Instrução sobre formas de relaxamento no pré-parto e parto & & & 0,01 \\
\hline Sim & 129 & 85,1 & \\
\hline Não & 85 & 72,1 & \\
\hline Alimentação durante permanência no centro obstétrico & & & 0,64 \\
\hline Sim & 26 & 75,6 & \\
\hline Não & 188 & 84,1 & \\
\hline Banho ou higienização no centro obstétrico & & & 0,64 \\
\hline Sim & 84 & 81,2 & \\
\hline Não & 130 & 78,3 & \\
\hline Tipo de parto & & & 0,01 \\
\hline Vaginal & 135 & 85,1 & \\
\hline Cesáreo & 79 & 71,4 & \\
\hline Toque vaginal foi feito por mais de um examinador & & & 0,86 \\
\hline Sim & 149 & 80,4 & \\
\hline Não & 65 & 80,3 & \\
\hline Foi feito episiotomia no parto & & & 0,73 \\
\hline Sim & 192 & 80,0 & \\
\hline Não & 22 & 82,4 & \\
\hline Teve acompanhante/familiar/amigos no centro obstétrico & & & 0,52 \\
\hline Sim & 11 & 73,3 & \\
\hline Não & 203 & 79,9 & \\
\hline Identificou as pessoas que prestaram atendimento & & & 0,50 \\
\hline Sim & 182 & 79,8 & \\
\hline Não & 29 & 80,6 & \\
\hline Não sabe & 3 & 60,0 & \\
\hline
\end{tabular}


Fatores associados ao fato das adolescentes considerarem que o trabalho desenvolvido pela equipe influenciou no parto (N=214). Rio Grande, RS, 2008-2009.

\begin{tabular}{lcc}
\hline Variável & $\mathbf{N}$ & $\boldsymbol{p}$ \\
\hline Recebeu o bebê assim que nasceu & & 0,26 \\
$\quad$ Sim & 142 & 81,8 \\
$\quad$ Não & 72 & 75,3 \\
Bebê mamou no centro obstétrico ou sala de recuperação & 192 & 80,6 \\
$\quad$ Sim & 22 & 75,6
\end{tabular}

*Teste exato de Fisher; **Critério de Classificação Econômica Brasil. São Paulo: ABEP= Associação Brasileira de Empresas de Pesquisa - 2007.

\section{Discussão}

Um dos aspectos preconizados pelas condutas ao parto e evocados em prol da humanização é a participação e o envolvimento da família nesse processo. ${ }^{6}$ A facilitação do acesso de um familiar de escolha da gestante ou do marido são elementos decisivos para o melhor prognóstico e andamento do parto, e está garantida pelo Estatuto da Criança e do Adolescente. 10

Porém, essa prática não vem sendo seguida pelo serviço em estudo, onde foi reduzido o índice de adolescentes que puderam contar com um acompanhante, e quando questionadas sobre o direito ao acompanhante durante os períodos de pré-parto e parto, observa-se que a noção e conhecimento de direitos são ainda pouco concretos para as usuárias dos serviços de saúde, principalmente quando dependem do SUS. 11

A quase totalidade das adolescentes relatou ter tido sua intimidade e privacidade respeitadas, o que se dá com o estabelecimento de relações baseadas em princípios éticos, garantindo-se assim a privacidade e compartilhando com a mulher e sua família as decisões sobre as condutas a serem adotadas. ${ }^{2}$

$\mathrm{O}$ fornecimento às adolescentes de todas as informações e explicações que desejarem vem ao encontro do preconizado pelo MS, que prioriza as relações de acolhimento e respeito às mulheres. Outros estudos também identificam que a comunicação, a troca de informações e a orientação às mulheres, assim como a interação com a equipe associam-se com a satisfação da assistência recebida no atendimento ao parto. ${ }^{11,12}$

Quanto às medidas de conforto, higiene e formas de relaxamento, observa-se que há um longo caminho a percorrer até que estas se tornem rotineiras a todos os atendimentos, pelas limitações da àrea física do CO. Durante o trabalho de parto, pela excessiva secreção vaginal, sangramento, perda de líquido amniótico e sudorese, a higiene da parturiente deve ser muito estimulada, e essas medidas estão ligadas ao alívio e controle da dor por meios não farmacológicos, como o banho de chuveiro e a deambulação.13,14

A tricotomia antes do parto foi uma prática utilizada, e os achados mostram também que esta parece já fazer parte da cultura das mulheres, pois mais da metade das pacientes já chegaram ao serviço tricotomizadas. Outros estudos também confirmam o alto uso desta prática.15,16 O baixo percentual de realização do enema mostra que esta é uma prática em desuso, o que está de acordo com as orientações do MS, como uma prática ineficaz. ${ }^{2}$

A episiotomia foi amplamente realizada. Essa prática é considerada prejudicial e ineficaz, sendo indicada somente em $10 \%$ a $15 \%$ dos casos, pois sendo um procedimento cirúrgico, envolve riscos e pode trazer inúmeras complicações para a recuperação do pós-parto, não devendo ser uma prática rotineira e justificada apenas pela paridade da mulher. ${ }^{17,18}$

$\mathrm{O}$ toque vaginal foi realizado com frequência e de forma expressiva por mais de um examinador, o que torna importante contextualizar, que o local da pesquisa onde foram realizadas as entrevistas, é um hospital universitário, apresentando a presença contínua de acadêmicos que se encontram em seu processo de ensino-aprendizagem.

Não foi identificado em que momento do trabalho de parto ocorreu, mas destaca-se que os toques vaginais subsequentes ao do diagnóstico 
devem ser feitos somente quando a gestante estiver na fase ativa do trabalho de parto, e evitado em presença de rotura de membranas, pois os riscos de infecção aumentam. ${ }^{13}$

Quanto à posição adotada no parto, chama atenção à predominância da posição litotômica, não tendo sido referenciado outras modalidades de posições que auxiliem no trabalho de parto. A OMS recomenda que as parturientes sejam estimuladas a adotar posições não supinas, durante o trabalho de parto e parto, pois estas estão associadas à redução do tempo do segundo período do parto, a redução da necessidade de partos assistidos, de episiotomias, e da sensação dolorosa na fase expulsiva e das alterações da frequência cardíaca fetal. 19

Outra prática muito utilizada foi a cateterização venosa profilática de rotina, o que não é recomendado, pois impede e limita os movimentos da puérpera e, por ser uma prática invasiva, pode representar riscos de infecção e traumas, além de aumentar a sensação de dor. ${ }^{7}$

A restrição hídrica e alimentar durante o trabalho de parto é rotina na instituição, mas, sabendo-se que a maioria das gestantes está em sua primeira gravidez, que o trabalho de parto pode levar em média 12 horas e que o gasto de energia é grande, essa restrição pode aumentar ainda mais o desconforto, podendo reduzir os níveis de glicemia e, dessa forma, acarretar riscos para o feto. Assim, a prática recomendada pelo MS é de avaliar a conduta com cautela e, para as gestantes de baixo risco, oferecer alimentos leves e líquidos durante a fase ativa do trabalho de parto. ${ }^{2}$

O tipo de parto predominante foi o vaginal, o que, segundo as adolescentes que o realizaram, foi o tipo de parto desejado, o que difere daquelas submetidas à cesárea, as quais relataram de forma expressiva não ser o que esperavam. Resultado semelhante foi encontrado por Bruzadeli e Tavares, ${ }^{20}$ em estudo de caso controle com mulheres adolescentes e adultas.

O incentivo ao contato precoce mãe/bebê e a amamentação ainda na primeira hora de vida ocorreram para a maioria dos binômios, possibilitando o fortalecimento do vínculo e o estímulo precoce ao aleitamento materno, conforme o preconizado pelo MS. ${ }^{2}$ Ainda assim, devem ser adotadas estratégias para que esse incentivo atinja a totalidade das pacientes. Os benefícios desta prática, vão desde a amamentação durar por mais tempo, a tendência materna em demonstrar afeto ao bebê, além de que, os bebês amamentados precocemente choraram por menos tempo. 21

O fato de que a realização do parto vaginal, a relação da equipe e o oferecimento de instruções sobre formas de relaxamento revelaram associação significativa com a influência positiva da equipe no trabalho de parto das adolescentes, vem ao encontro do preconizado pelo MS, que prioriza o parto vaginal e as relações de acolhimento e respeito às mulheres. Outros estudos também identificam que a comunicação, a troca de informações e a orientação às mulheres, assim como a interação com a equipe associam-se com a satisfação da assistência recebida no atendimento ao parto. ${ }^{15}$

A qualidade da relação com a equipe, o respeito à privacidade e à intimidade somada ao oferecimento de instruções de relaxamento para o parto estarem associados com o atendimento considerado ideal pelas adolescentes, provavelmente indicam o quanto a qualidade das relações humanas é importante para essas jovens, tornando-se condutas relevantes para o atendimento ao parto.

Ser adolescente e conviver com um parto neste momento de sua vida assume um sentido positivo ou negativo, de acordo com a experiência vivida. Tocála em demasia, mantê-la em posição litotômica, restringir líquidos e alimentos, submetê-la à episiotomia e à cateterização venosa de rotina são exemplos de condutas que além de irem contra as orientações do MS, ainda podem ser traumáticas para uma adolescente. Um estudo desenvolvido em Belo Horizonte (MG) com adolescentes ressalta o importante papel da equipe de saúde na atenção ao parto, tendo sido visualizado que alguns procedimentos realizados com as adolescentes fez com que estas se sentissem agredidas e submissas. 22

Quanto à presença de algumas práticas consideradas prejudiciais pelo MS e que não são recomendadas terem aparecido com relevada significância no presente estudo, como a cateterização venosa profilática de rotina, a restrição hídrica, e a episiotomia, podemos atribuir ao fato da instituição pesquisada ser um hospital de ensino, com residência médica em obstetrícia, podendo demonstrar que o uso destas técnicas, dão (falsa) "segurança" aos profissionais em caso de alguma intercorrência.

O uso da cateterização pode estar sendo utilizado pelo risco de hemorragias e o receio de precisar proceder a esse tipo de intervenção em um momento de urgência; a restrição hídrica pode estar sendo pensada pelo risco de uma cesariana de urgência; a episiotomia como forma de evitar lacerações no períneo, pelo próprio aprendizado, e associada a insegurança de que este procedimento realmente não se faz necessário.

O estudo pode assim demonstrar algumas limi- 
tações, por se tratar de uma pesquisa desenvolvida em um hospital universitário, que tem uma dinâmica diferenciada de outras instituições.

Avaliar a atenção ao parto pela perspectiva do usuário (adolescente) permitiu não somente avaliar as condutas e rotinas adotadas no serviço e comparálas com o preconizado pela OMS/MS em relação ao parto normal, mas também refletir sobre as relações interpessoais na assistência ao parto e no processo de cuidar, em parturientes adolescentes.

Porém, quanto às condutas preconizadas e que ainda não foram totalmente incorporadas ao serviço, sugere-se aos gestores e à equipe multidisciplinar de atendimento ao parto programar ações que possibilitem atingir o que é preconizado, por meio da realização de cursos de atualização, ou educação

\section{Referências}

1. Gurgel MGI, Alves MDS, Vieira NFC, Pinheiro PNC, Barroso GT. Gravidez na adolescência: tendência na produção científica de enfermagem. Esc Anna Nery Rev Enferm. 2008; 12: 800-6

2. Brasil. Ministério da Saúde. Secretaria de Atenção à Saúde. Departamento de Ações Programáticas Estratégicas. Área Técnica de Saúde da Mulher. Pré-natal e puerpério: atenção qualificada e humanizada - manual técnico/Ministério da Saúde, Secretaria de Atenção à Saúde, Departamento de Ações Programáticas Estratégicas. Brasília, DF; 2005.

3. Gonçalves CV, Cesar JA, Sassi RAM. Qualidade e equidade na assistência à gestante. Cad Saúde Pública. 2009; 25 . 2507-16.

4. Spindola AT, Silva LFF. Perfil epidemiológico de adolescentes atendidas no pré-natal de um hospital universitário. Esc Anna Nery Rev Enferm. 2009; 13: 99-107.

5. Oliveira MIC, Dias MAB, Cunha CB, Leal MC. Qualidade da assistência ao trabalho de parto pelo Sistema Único de Saúde (RJ), 1999-2001. Rev Saúde Pública. 2008; 42: 895902.

6. Brasil. Portaria GM/MS n ${ }^{\circ} 569$, de 01 de junho de 2000 Institui o Programa de Humanização no Pré-Natal e Nascimento no âmbito do Sistema Único de Saúde. Diário Oficial da União. Brasília, DF 1 jun 2000

7. (OMS) Organização Mundial da Saúde. Assistência ao parto normal: um guia prático. Relatório de um grupo técnico. Genebra; 1996. 53 p.

8. Teixeira GG, Chanes M. As estratégias de humanização da assistência ao parto utilizadas por hospitais ganhadores do Prêmio Galba de Araújo: ações de mérito, ações premiadas. Mundo Saúde. 2003; 27: 270-3.

9. Manzini FC, Borges VTM, Parada CMGL. Avaliação da assistência ao parto em maternidade terciária do interior do Estado de São Paulo, Brasil. Rev Bras Saúde Matern Infant. 2009; 9: 59-67.

10. Brasil. Estatuto da Criança e do Adolescente (ECA). Brasília, DF; 1990 continuada.

$\mathrm{O}$ uso das práticas recomendadas pela OMS/MS ao parto abre a possibilidade de se criar novas maneirar de vivenciar a maternidade, recuperando o protagonismo da mulher. Com o compromisso de todas as partes envolvidas, gestores de saúde, equipe multidisciplinar e setores de apoio, é possível visualizar a retomada do parto como um acontecimento mais humano e familiar.

A atenção ao parto se dá com uma equipe cuidadosa e atenta durante o trabalho de parto e parto, oferecendo instruções sobre formas de relaxamento, respeitando a intimidade e privacidade. Atitudes, que associadas a qualidade da relação com a equipe do cento obstétrico fizeram a diferença para as adolescentes do estudo.
11. Merighi MAB, Carvalho MG, Suletroni VP. O processo de parto e nascimento: visão das mulheres que possuem convênio saúde na perspectiva da fenomenologia social. Acta Paul Enferm. 2007;20: 434-40. Disponível em: http://www.scielo.br/pdf/ape/v20n4/07.pdf

12. Rodrigues AV, Siqueira AAF. Sobre as dores e temores do parto: dimensões de uma escuta. Rev Bras Saúde Matern Infant. 2008; 8: 176-86.

13. Brasil. Ministério da Saúde. Secretaria de Políticas de Saúde. Área Técnica da Saúde da Mulher. Parto, aborto e puerpério: assistência humanizada à mulher. Brasília, DF; 2001.

14. Davim RMB, Torres GV, Dantas JC, Melo ES, Paiva CP, Vieira D, Costa IKF. Banho de chuveiro como estratégia não farmacológica no alívio da dor de parturientes. Rev Eletr Enf. 2008; 10: 600-9. Disponível em: http://www.fen.ufg.br/revista/v10/n3/v10n3a06.htm

15. D’Orsi E, Chor D, Giffin K, Ângulo-Tuesta A, Barbosa GP, Gama AS, Reis AC, Hartz Z. Qualidade da atenção ao parto em maternidades do Rio de Janeiro. Rev Saúde Pública. 2005; 39: 646-54.

16. Sodré TM, Lacerda RA. O processo de trabalho na assistência ao parto em Londrina-PR. Rev Esc Enferm USP. 2007; 41: 82-9.

17. Romero KT, Medeiros EHGR, Vitalle MSAS, Wehba JO. Conhecimento das dolescentes sobre questões relacionadas ao sexo. Rev Assoc Med Bras. 2007; 53: 14-7.

18. Rodriguez LF, Arias MI, Charry RC, Blasco de OM, Rivas AM, Brucoli GV. Episiotomia selectiva versus rutinaria em pacientes nulíparas. Rev Obst Ginecol Venez. 2004; 64: 121-7.

19. Cecatti J, Calderón IMP. Intervenções benéficas durante o parto para a prevenção da mortalidade materna. Rev Bras Ginecol Obst. 2005; 27: 357-65. 
20. Bruzadeli DS, Tavares BB. Expectativa quanto ao parto e conhecimento do motivo da cesárea entre puérperas adolescentes e adultas. Rev Eletr Enf. 2010; 12: 150-7.

21. Anderson GC, Moore E, Hepworth J, Bergman N. Early skin to skin contact for mothers and their healthy newborn infants. In: Cochrane Database of Systematic Reviews, Issue 3, 2007.

Recebido em 21 de julho de 2011

Versão final apresentada em 30 de agosto de 2012

Aprovado em 27 de setembro de 2012
22. Oliveira ZMLP, Madeira AMF. Vivenciando o parto humanizado: um estudo fenomenológico sob a ótica de adolescentes. Rev Esc Enferm USP. 2002; 36: 133-40. 